Research Article

\title{
Nonparametric Blind Signal Detection Based on Logarithmic Moments in Very Impulsive Noise
}

\author{
Jinjun Luo $\mathbb{D},{ }^{1}$ Shilian Wang $\mathbb{D},{ }^{1}$ Eryang Zhang, ${ }^{1}$ and Xin Man $\mathbb{D}^{2}$ \\ ${ }^{1}$ School of Electronic Science and Engineering, National University of Defense Technology, Changsha 410073, China \\ ${ }^{2}$ College of Electronic Engineering, Naval University of Engineering, Wuhan 430033, China \\ Correspondence should be addressed to Jinjun Luo; jinjunluo@outlook.com
}

Received 2 October 2017; Accepted 5 March 2018; Published 12 April 2018

Academic Editor: Jinglan Zhang

Copyright (c) 2018 Jinjun Luo et al. This is an open access article distributed under the Creative Commons Attribution License, which permits unrestricted use, distribution, and reproduction in any medium, provided the original work is properly cited.

The detection problem in impulsive noise modeled by the symmetric alpha stable $(\mathrm{S} \alpha \mathrm{S})$ distribution is studied. The traditional detectors based on the second or higher order moments fail in S $\alpha$ S noise, and the method based on the fractional lower order moments (FLOMs) performs poorly when the noise distribution has small values of characteristic exponent. In this paper, a detector based on the logarithmic moments is investigated. The analytical expressions of the false alarm and detection probabilities are derived in nonfading channels as well as Rayleigh fading channels. The effect of noise uncertainty on the performance is discussed. Simulation results show that the logarithmic detector performs better than the FLOM and Cauchy detectors in very impulsive noise. In addition, the logarithmic detector is a nonparametric method and avoids estimating the parameter of the noise distribution, which makes the logarithmic detector easier to implement than the FLOM detector.

\section{Introduction}

Signal detection in noise is a classical problem in signal processing, and it is a key technology for mobile information systems. Usually, mobile information systems are designed under the assumption of Gaussian noise for mathematical convenience. However, in many applications, the background noise is non-Gaussian and shows impulsive behavior, such as man-made noise, interference from adjacent channel, and radio frequency interference (RFI) [1-3]. In impulsive noise, the conventional detection methods optimized under the Gaussian noise assumption suffer from a drastic performance degradation, which degrades the overall performance of the mobile information systems. Thus, more accurate models for impulsive noise are developed. The generalized Gaussian (GG) distribution, Gaussian mixture (GM) model, Middleton Class-A (MCA) model, and symmetric alpha stable $(S \alpha S)$ distribution are alternative models for impulsive noise.

The $S \alpha S$ distribution has a solid theoretical foundation that it satisfies the generalized central limit theorem and has an advantage of stability. In addition, empirical data prove that the $S \alpha S$ distribution is a successful model for impulsive noise in mobile information systems. For example, radio frequency interference (RFI) for the embedded wireless data transceivers can be modeled using symmetric alpha stable $(\mathrm{S} \alpha \mathrm{S})$ distributions with a characteristic exponent of 1.43 [2]. The co-channel interference in a communication link in a Poison field of interferers shows very impulsive behavior and can be modeled using $S \alpha S$ distributions with small characteristic exponent values [4]. In medical diagnosis or mobile health data processing, evoked potential (EP) detection is an important problem. Experimental analysis showed that the noised EP signal could be successfully modeled using $\mathrm{S} \alpha \mathrm{S}$ distributions with characteristic exponent values between 1.06 and 1.94 [5]. In [6], the noise fitting procedure indicated that the $S \alpha S$ distribution could fit the noise from GSM-R antennas much better than the Middleton Class-A model and Gaussian distributions. Due to these advantages, the $S \alpha S$ distribution has received an increasing attention for modeling the impulsive noise in recent years. In this paper, we consider the signal detection problem in very impulsive $S \alpha S$ distributed noise.

In the early literature, the proposed detectors for $S \alpha S$ noise usually require prior knowledge of the transmitted signal. This kind of detectors mainly includes the optimal detector and the locally optimal detector under a weak signal assumption. As the probability density function (PDF) of 
S $\alpha$ S has no closed form expression, the optimal and locally optimal detectors should be implemented through numerical methods, which results in a heavy computational burden. Thus, some locally suboptimal detectors are proposed, such as the soft limiter, hole puncher, and local Cauchy detector [3]. Some detectors based on the approximate expression for the PDF of the $S \alpha S$ distribution are also investigated $[7,8]$.

In some practical applications, such as the communication countermeasure, and cognitive radio, the transmitted signal is not available. The aforementioned detectors cannot be used directly, and thus blind or semiblind detection techniques are required. The generalized likelihood ratio test (GLRT) is an optimal solution, but it is extremely timeconsuming [9]. The blind Cauchy detector is a special case of the GLRT and only performs well for some characteristic exponent in $S \alpha S$ noise. Recently, a scheme based on the fractional lower order moment (FLOM) is investigated in [10]. It performs better than the blind Cauchy detector. However, when the noise distribution has small values of the characteristic exponent, the FLOM detector performs poorly. In addition, the moment order of the FLOM should be determined, whose choice depends on the estimated characteristic exponent.

The logarithmic process was first introduced in [11]. Then the logarithmic moment (LM) was utilized for parameter estimation of the $S \alpha S$ distribution [12-14]. However, its application to signal detection has not yet received much attention. In this paper, we apply the logarithmic moment to signal detection and propose a detector based on it. The performance of the logarithmic detector is investigated in detail. The main contributions include the following: (i) We derive the analytical expressions of the false alarm and detection probabilities in nonfading channels as well as Rayleigh fading channels. (ii) The effect of the noise uncertainty on the detection probability is discussed, and the detection performance with different characteristic exponents and sample sizes is also investigated. (iii) The simulation results illustrate that the logarithmic detector performs better than the Cauchy and FLOM detectors when the characteristic exponent is smaller than 1.5. (iv) The logarithmic detector is a nonparametric method and avoids estimating the parameter of the noise distribution, which make the logarithmic detector easier to implement than the FLOM detector.

The remainder of this paper is organized as follows. In Section 2, the system and noise models are presented and the conventional detectors are introduced. In Section 3, the logarithmic detector is proposed and its performance is analyzed. In Section 4, extensive simulations are carried out. Finally, conclusions are drawn in Section 5.

\section{System Model and Conventional Detectors}

2.1. System Model. In this paper, the word "signal detection" refers to deciding whether a signal is present or not in the received data. It can be formulated as a binary hypothesis testing problem, described by $H_{0}$ : signal absent and $H_{1}$ : signal present. It can be written as

$$
\begin{aligned}
& H_{0}: z(n)=w(n), \\
& H_{1}: z(n)=h s(n)+w(n),
\end{aligned}
$$

where $z(n)$ is the received data sample at time $n \in$ $\{1,2, \ldots, N\}, w(n)$ represents the impulsive noise, $s(n)$ is the transmitted signal, and $h$ is the channel gain between the transmitter and receiver.

The impulsive noise $w(n)$ is modeled by an independent and identically distributed (i.i.d.) $\mathrm{S} \alpha \mathrm{S}$ distribution. Since the received signal is usually a sum of multiple nonline of sight (NLOS) signals, $s(n)$ can be modeled as a Gaussian random variable with zero mean according to the central limit theorem and is assumed to be independent of the noise $w(n)$.

The $S \alpha S$ distribution is described by its characteristic function:

$$
\varphi_{\alpha}(t)=\exp \left(-\gamma|t|^{\alpha}\right),
$$

where $0<\alpha \leq 2$ and $\gamma>0 . \alpha$ is the characteristic exponent and determines the thickness of the distribution tail. When $\alpha$ is smaller, the tail is more thick and the pulse characteristic is more noticeable. $\gamma$ is the scale parameter, also known as the dispersion parameter. It indicates the degree of the dispersion spread which is similar to the variance in a Gaussian distribution.

The PDF of the S $\alpha$ S distribution is the inverse Fourier transform of the characteristic function, namely,

$$
f_{\alpha}(x)=\frac{1}{2 \pi} \int_{-\infty}^{\infty} \varphi_{\alpha}(t) e^{-j t x} d x .
$$

The PDF does not have a closed form expression except for $\alpha=2$ and $\alpha=1$. In the two special cases, the $S \alpha S$ distribution reduces to Gaussian distribution and Cauchy distribution, respectively.

2.2. Conventional Detectors. In blind signal detection, the transmitted signal and channel gain are usually not available, so the likelihood ratio test (LRT) cannot be used directly. We can first estimate the unknown parameters and then apply the likelihood ratio test, which is known as the generalized likelihood ratio test, defined as [9]

$$
\Lambda_{\mathrm{GLRT}}=\sum_{n=1}^{N} \log \frac{f_{\alpha}(z(n)-\hat{h} \hat{s}(n))}{f_{\alpha}(z(n))},
$$

where $\hat{h}$ and $\hat{s}(n)$ are the maximum likelihood estimates of $h$ and $s(n)$. Due to the numerical evaluation of $f_{\alpha}(x)$ and the parameter estimation, the GLRT detector is too complex to be applied for real-time applications. In the special case of $\alpha=1$, the GLRT detector becomes a Cauchy detector [9], namely,

$$
\Lambda_{\text {Cauchy }}=\sum_{n=1}^{N} \log \left\{1+\frac{|z(n)|^{2}}{\gamma^{2}}\right\} .
$$

In the case of $0<\alpha<2$, the second and higher order moments do not exist, so the traditional detector based on these moments cannot be used. However, the fractional lower moments (FLOMs) of any order less than $\alpha$ do exist. Then, the FLOM detector is proposed [10], which is defined as 


$$
\Lambda_{\mathrm{FLOM}}=\frac{1}{N} \sum_{n=1}^{N}|z(n)|^{p},
$$

where $p$ is the order of the fractional moment. In order to ensure the existence of the FLOM statistic variance, the allowable value of $p$ should be limited between 0 and $\alpha / 2$. The choice of $p$ depends on $\alpha$ which could be estimated by some methods $[12,13]$.

\section{Nonparametric Logarithmic Detector and Performance Analysis}

3.1. Logarithmic Moment-Based Detector. The logarithmic moment has been successfully applied to parameter estimation of the $\mathrm{S} \alpha \mathrm{S}$ distribution, but few studies investigate its application to signal detection. In this paper, we proposed a detector based on the logarithmic moment (LM). The LM detector is defined as

$$
\Lambda_{\mathrm{LM}}=\frac{1}{N} \sum_{i=1}^{N} \log |z(n)| \underset{H_{0}}{\stackrel{H_{1}}{\gtrless}} \eta,
$$

where $\eta$ is the detection threshold which could be determined by a given $P_{\mathrm{f}}$. Compared with the FLOM detector, this detector does not need to decide the moment order $p$, which is convenient in practical applications.

\subsection{Performance Analysis of the Logarithmic Detector in} Nonfading Channels. The statistic $\Lambda_{\mathrm{LM}}$ is the sum of $N$ independent random variables. When $N$ is large enough, the statistic follows an asymptotic Gaussian distribution under either hypothesis due to the central limit theorem. To calculate the false alarm and detection probabilities, the mean and variance of the statistic should be calculated first.

Under hypothesis $H_{0}$, the mean and variance of $\Lambda_{\mathrm{LM}}$ can be calculated as (see Appendix for detail)

$$
\begin{aligned}
& u_{0}=E\left\{\Lambda_{\mathrm{LM}} \mid H_{0}\right\}=C_{e}\left(\frac{1}{\alpha}-1\right)+\frac{1}{\alpha} \log \gamma, \\
& \sigma_{0}^{2}=E\left\{\Lambda_{\mathrm{LM}}^{2} \mid H_{0}\right\}-E^{2}\left\{\Lambda_{\mathrm{LM}} \mid H_{0}\right\}=\frac{\pi^{2}}{6 N}\left(\frac{1}{\alpha^{2}}+\frac{1}{2}\right) .
\end{aligned}
$$

Under hypothesis $H_{1}$, the mean of $\Lambda_{\mathrm{LM}}$ can be expressed as

$$
\begin{aligned}
u_{1}=E\left\{\Lambda_{\mathrm{LM}} \mid H_{1}\right\}= & -\int_{0}^{\infty} E(u) \ln (u) F(u) d u \\
& -C_{e} * \int_{0}^{\infty} E(u) F(u) d u,
\end{aligned}
$$

where

$$
\begin{aligned}
E(u) & =\exp \left(-\gamma u^{\alpha}-\frac{h^{2} \sigma_{s}^{2}}{2} u^{2}\right), \\
F(u) & =\gamma \alpha u^{\alpha-1}+h^{2} \sigma_{s}^{2} u, \\
C_{e} & =0.577215 \ldots
\end{aligned}
$$

The variance of $\Lambda_{\mathrm{LM}}$ can be computed as

$$
\begin{aligned}
\sigma_{1}^{2} & =\frac{1}{N}\left\{E\left[(\log |z|)^{2}\right]-(E(\log |z|))^{2}\right\} \\
& =\frac{1}{N}\left\{E\left[(\log |z|)^{2}\right]-u_{1}^{2}\right\},
\end{aligned}
$$

where

$$
\begin{aligned}
E\left[(\log |z|)^{2}\right]= & \int_{0}^{\infty} E(u)(\ln (u))^{2} F(u) d u \\
& +2 C_{e} \int_{0}^{\infty} E(u) \ln (u) F(u) d u \\
& +\left(C_{e}^{2}+\frac{\pi^{2}}{12}\right) \int_{0}^{\infty} E(u) F(u) d u
\end{aligned}
$$

Then the false alarm and detection probabilities can be calculated as

$$
\begin{aligned}
& P_{\mathrm{f}}=\left\{\Lambda_{\mathrm{LM}}>\eta \mid H_{0}\right\}=Q\left(\frac{\eta-\mu_{0}}{\sigma_{0}}\right), \\
& P_{\mathrm{d}}=\left\{\Lambda_{\mathrm{LM}}>\eta \mid H_{1}\right\}=Q\left(\frac{\eta-\mu_{1}}{\sigma_{1}}\right) .
\end{aligned}
$$

The expression of $P_{\mathrm{f}}$ has a closed form, so the threshold $\eta$ can be easily calculated from this expression, where the parameters $\alpha$ and $\gamma$ can be estimated by many methods $[12,13]$. Although the expression of $P_{d}$ has no closed form, it can be evaluated by numerical methods. Through evaluating the expression of $P_{\mathrm{d}}$, we can conveniently obtain the relationship between the detection probability and the sample size under different GSNRs, which gives us useful guideline for the choice of the sample size in practical applications.

In the following, we consider the effect of noise uncertainty on the logarithmic detector. Noise uncertainty is mainly caused by temperature variation, nonlinearity of components, initial calibration error, and interference $[15,16]$. In general, a detector with a fixed threshold is designed based on the nominal noise power which is estimated in a finite time, so the performance will degrade when the actual noise power does not equal the nominal noise power.

From (8), (9), and (16), the threshold for logarithmic detector depends on the knowledge of the parameters $\gamma$ and $\alpha$ which should be estimated by taking a large number of samples but some residual uncertainty still exists. Next, we only analyze the effect of uncertainty of $\gamma$ on the detection performance; a similar procedure can be carried out for the parameter $\alpha$.

We denote the nominal noise dispersion or the estimated noise dispersion by $\gamma_{\mathrm{n}}$ and the actual noise dispersion by $\gamma_{\mathrm{a}}$. In the presence of noise uncertainty, $\gamma_{\mathrm{a}}=\rho \gamma_{\mathrm{n}}$, where $\rho$ is the noise uncertainty coefficient, and its value in $\mathrm{dB}$ is denoted by $\beta=10 \log _{10} \rho$. Usually, $\beta$ is uniformly distributed in the interval $[-B, B][10,17]$. Then, the PDF of $\beta$ can be expressed as

$$
f(\beta)= \begin{cases}\frac{1}{2 B}, & -B<\beta<B \\ 0, & \text { otherwise. }\end{cases}
$$


According to the relationship between $\gamma_{\mathrm{a}}$ and $\beta$, we can easily obtain the PDF of $\gamma_{\mathrm{a}}$ as follows:

$$
f\left(\gamma_{\mathrm{a}}\right)= \begin{cases}\frac{10}{2 B \gamma_{\mathrm{a}} \ln 10}, & \gamma_{\mathrm{n}} 10^{-B / 10}<\gamma_{\mathrm{a}}<\gamma_{\mathrm{n}} 10^{B / 10} \\ 0, & \text { otherwise. }\end{cases}
$$

In nonpresence of noise uncertainty, the false alarm and detection probabilities can be calculated using (16) and (17). When the noise uncertainty does exist, the noise dispersion $\gamma$ is no longer a constant value. In this case, we view (16) and (17) as conditional probabilities, where the condition is that $\gamma$ is fixed. Then $P_{\mathrm{f}}$ and $P_{\mathrm{d}}$ are functions of the actual noise dispersion $\gamma_{\mathrm{a}}$, and they can be rewritten as $P_{\mathrm{f}}\left(\gamma_{\mathrm{a}}\right)$ and $P_{\mathrm{d}}\left(\gamma_{\mathrm{a}}\right)$, respectively. By averaging (16) and (17) over (19), the probabilities of false alarm and detection under noise uncertainty can be obtained as

$$
\begin{aligned}
& \bar{P}_{\mathrm{f}}=\int_{b}^{c} P_{\mathrm{f}}\left(\gamma_{\mathrm{a}}\right) f\left(\gamma_{\mathrm{a}}\right) d \gamma_{\mathrm{a}}, \\
& \bar{P}_{\mathrm{d}}=\int_{b}^{c} P_{\mathrm{d}}\left(\gamma_{\mathrm{a}}\right) f\left(\gamma_{\mathrm{a}}\right) d \gamma_{\mathrm{a}},
\end{aligned}
$$

where $b=\gamma_{\mathrm{n}} 10^{-B / 10}$ and $c=\gamma_{\mathrm{n}} 10^{B / 10} \cdot \bar{P}_{\mathrm{f}}$ and $\bar{P}_{\mathrm{d}}$ do not have a closed form expression, but they can be evaluated by numerical methods.

3.3. Performance Analysis of the Logarithmic Detector in Rayleigh Fading Channels. In Rayleigh fading channels, $P_{\mathrm{f}}$ remains the same as that of (16) in nonfading channels. The average detection probability can be evaluated by averaging the conditional $P_{\mathrm{d}}$ in the nonfading case as given in (17) over the channel gain. In this case, $P_{\mathrm{d}}$ in (17) can be regarded as a function of $h$, rewritten as $P_{\mathrm{d}}(D \mid h)$. The average detection probability $\bar{P}_{\mathrm{d}}$ in Rayleigh fading channels can be obtained as follows:

$$
\bar{P}_{\mathrm{d}}=E_{h}\left[P_{\mathrm{d}}(D \mid h)\right]
$$

Since the channel gain $h$ is Rayleigh distributed, its PDF is shown as

$$
f(h)=\frac{h}{\sigma^{2}} \exp \left(-\frac{h^{2}}{2 \sigma^{2}}\right), \quad h \geq 0,
$$

where $\sigma^{2}=\sqrt{2 / \pi} E[h]$. So, $\bar{P}_{\mathrm{d}}$ can be calculated as

$$
\begin{aligned}
\bar{P}_{\mathrm{d}} & =\int_{0}^{\infty} P_{\mathrm{d}}(D \mid h) f(h) d h \\
& =\int_{0}^{\infty} \mathrm{Q}\left(\frac{\eta-\mu_{1}}{\sigma_{1}}\right) f(h) d h .
\end{aligned}
$$

It is difficult to find a closed form expression for $\bar{P}_{\mathrm{d}}$, but we can evaluate it by numerical methods. Specially, when the GSNR is low enough where $\sigma_{s}^{2} \approx 0$ and $\sigma_{1}^{2} \approx \sigma_{0}^{2}, \bar{P}_{\mathrm{d}}$ can be simplified as

$$
\bar{P}_{\mathrm{d}}=\int_{0}^{\infty} \mathrm{Q}\left(\frac{\eta-\mu_{1}}{\sigma_{0}}\right) f(h) d h .
$$

Because $\sigma_{0}^{2}$ is not a function of $h$, the integral will be greatly simplified.

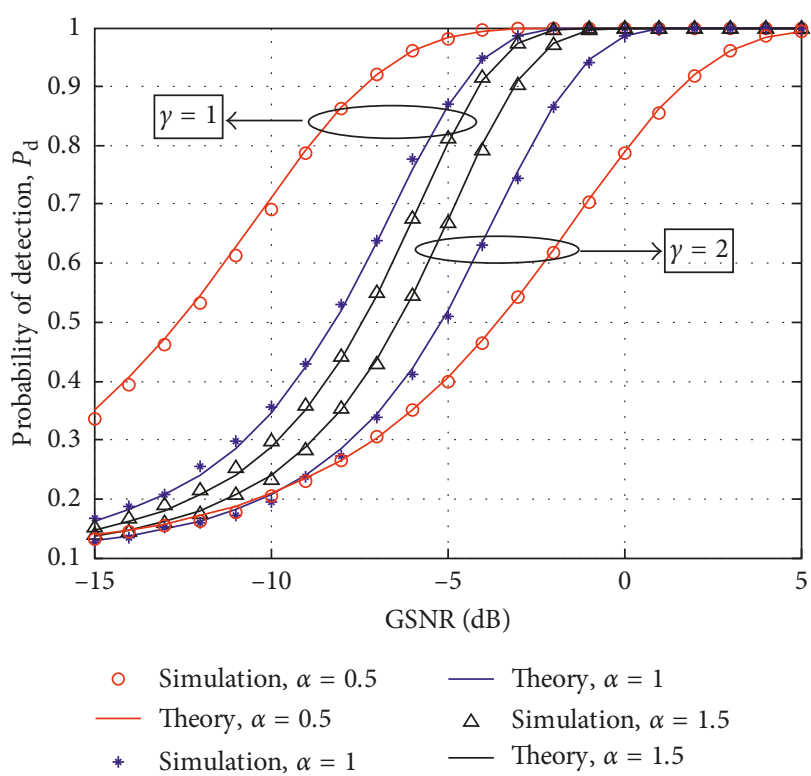

FIgURE 1: Detection probability versus GSNR for the logarithmic detector, $P_{\mathrm{f}}=0.1$, and $N=1000$.

\section{Simulation Results and Analysis}

In this section, simulation results are presented to show the performance of the logarithmic detector. The channel is assumed fading free, unless otherwise specified. The transmitted signal is a Gaussian process with zero-mean and variance $\sigma_{s}^{2}=E\left[|s(n)|^{2}\right]$. The results are based on 10000 Monte-Carlo simulations with a generalized signal-to-noise ratio (GSNR) defined as GSNR $=10 \log _{10}\left(\sigma_{s}^{2} \sigma_{h}^{2} / \gamma\right)$ [10].

Figure 1 shows the detection probability versus the GSNR for the logarithmic detector in nonfading channels, where $P_{\mathrm{f}}=0.1$ and $N=1000$. The curves are obtained from the simulation using (7) and by evaluating the analytical expressions in (16) and (17). The theoretical results are consistent with the Monte-Carlo simulation results very well for different $\alpha$ and $\gamma$, which proves the accuracy of the analytical expressions of the false alarm and detection probabilities. In addition, for a fixed false alarm probability, the detection probability increases with the increment of $\alpha$ when $\gamma=2$, whereas it decreases with the increment of $\alpha$ when $\gamma=1$. Therefore, the relationship between the detection probability and $\alpha$ depends on the values of $\gamma$.

Figure 2 shows the detection probability versus characteristic exponent $\alpha$ for different values of the GSNR and dispersion parameter $\gamma$, where $P_{\mathrm{f}}=0.1$ and $N=1000$. The relationship between the detection probability and $\alpha$ is not definite, and it depends on the values of the GSNR and $\gamma$. For a fixed $\alpha$ and GSNR, the detection probability increases with the decrement of $\gamma$. When GSNR $=-8 \mathrm{~dB}$ and $\gamma=1$, the detection probability decreases with the increment of $\alpha$. This result indicates that the detection performance is better in the presence of increased impulsive noise, which opposites researchers' intuition that more impulsive noise results in worse detection performance. This intuition is one-sided and based on an assumption that the detection performance is determined by the background noise. In fact, the detection 




Figure 2: Detection probability versus $\alpha$ of the logarithmic detector, $P_{\mathrm{f}}=0.1$, and $N=1000$.

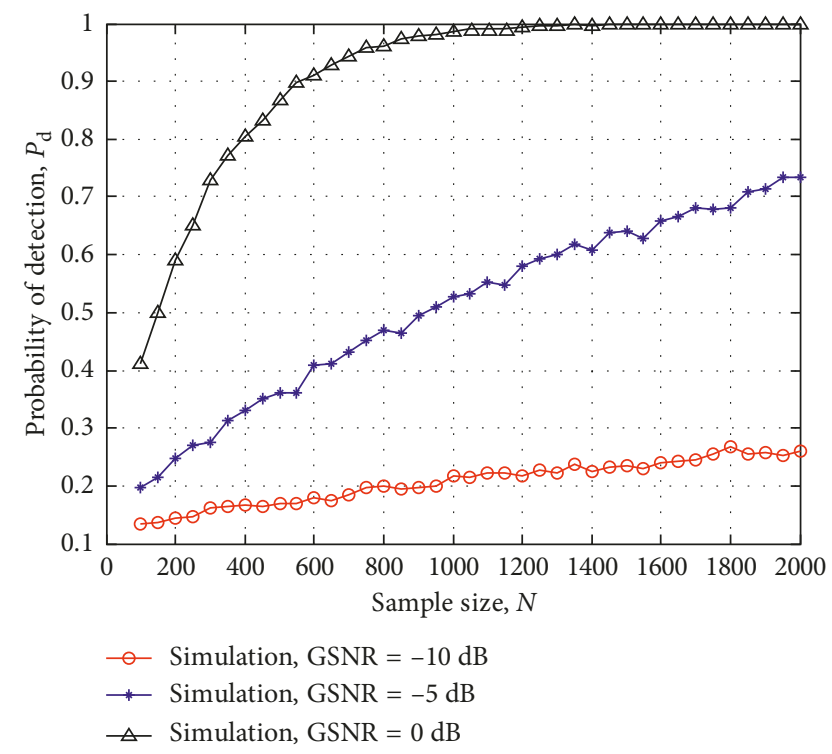

FIGURE 3: Detection probability versus number of samples for the logarithmic detector, $\alpha=1, \gamma=2$, and $P_{\mathrm{f}}=0.1$.

performance depends not only on the background noise but also on the detector. This special result indicates that the logarithmic detector has a good ability to deal with the impulsive noise.

Figure 3 shows the relationship between the detection probability and the sample size $N$, where $\alpha=1, \gamma=2$, and $P_{\mathrm{f}}=0.1$. The detection probability increases rapidly with the increment of $N$ under a high GSNR, whereas it increases slowly with the increment of $N$ under a low GSNR. Thus, when the GSNR is low, we should collect much more sample data to achieve a desired detection probability.

Figure 4 shows the receiver operation characteristic (ROC) curves of the logarithmic detector with different values of

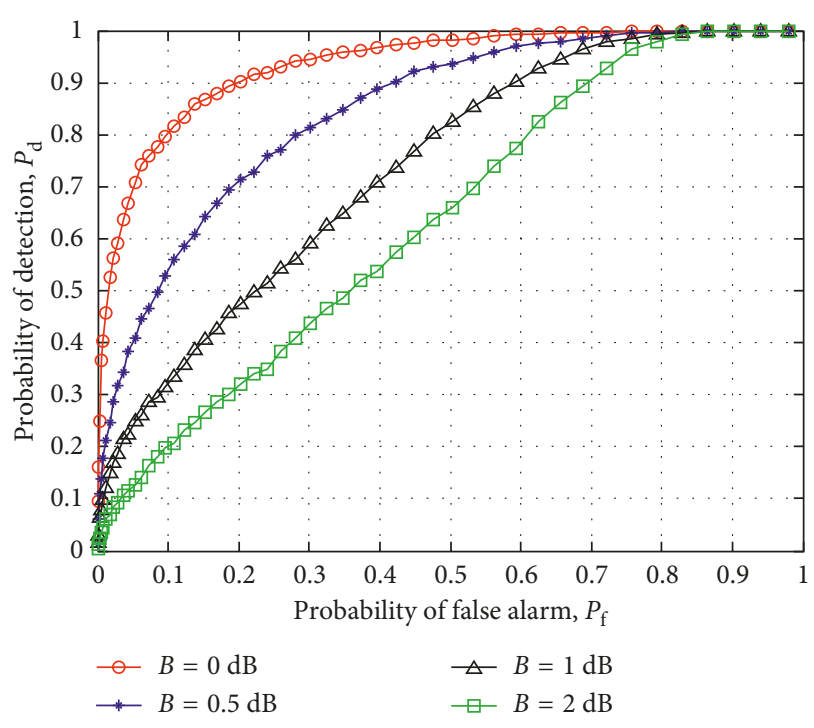

FIGURE 4: ROC of the logarithmic detector with noise uncertainty, $\alpha=1.5, \gamma=1, \mathrm{GSNR}=-5 \mathrm{~dB}$, and $N=1000$.

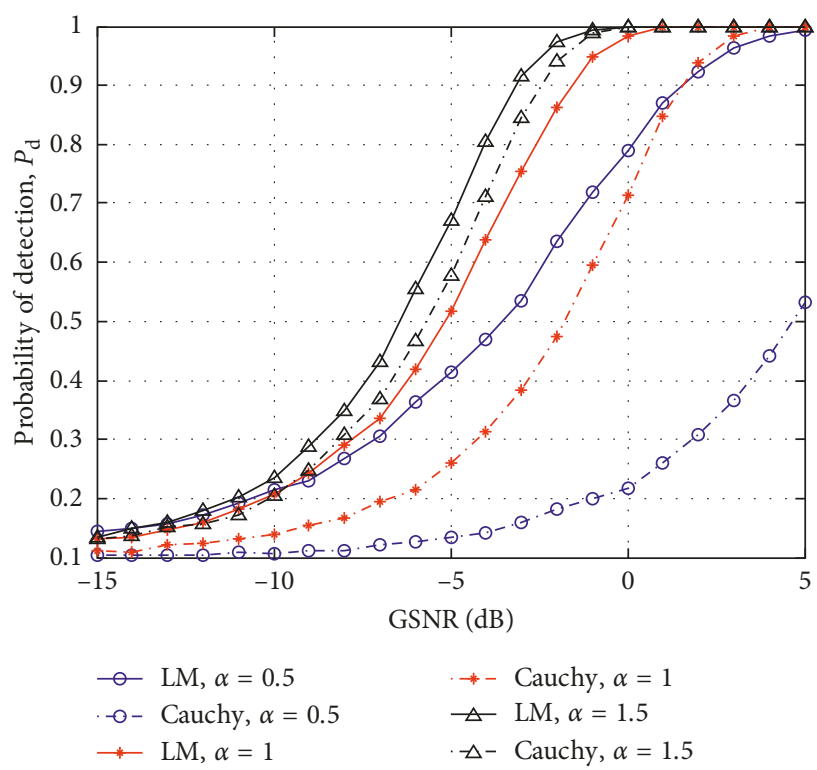

FIGURE 5: Detection probability versus GSNR for the logarithmic and Cauchy detectors in nonfading channels, $\gamma=2, P_{\mathrm{f}}=0.1$, and $N=1000$.

noise uncertainty, where $\alpha=1.5, \gamma=1$, GSNR $=-5 \mathrm{~dB}$, and $N=1000$. From this figure, we can see that the detection probability decreases with the increment of the noise uncertainty. When the value of $B$ increases from $0 \mathrm{~dB}$ to $2 \mathrm{~dB}$, the detection probability at $P_{\mathrm{f}}=0.1$ decreases from 0.8 to 0.2 . To mitigate the effect of noise uncertainty, cooperative detection techniques are presented in the literature [18]. In addition, more accurate methods should be adopted to estimate the parameters $\alpha$ and $\gamma$ based on more sample data.

Figure 5 compares the detection probability between the logarithmic and Cauchy detectors in nonfading channels, where $\gamma=2, P_{\mathrm{f}}=0.1$, and $N=1000$. We can see that the 

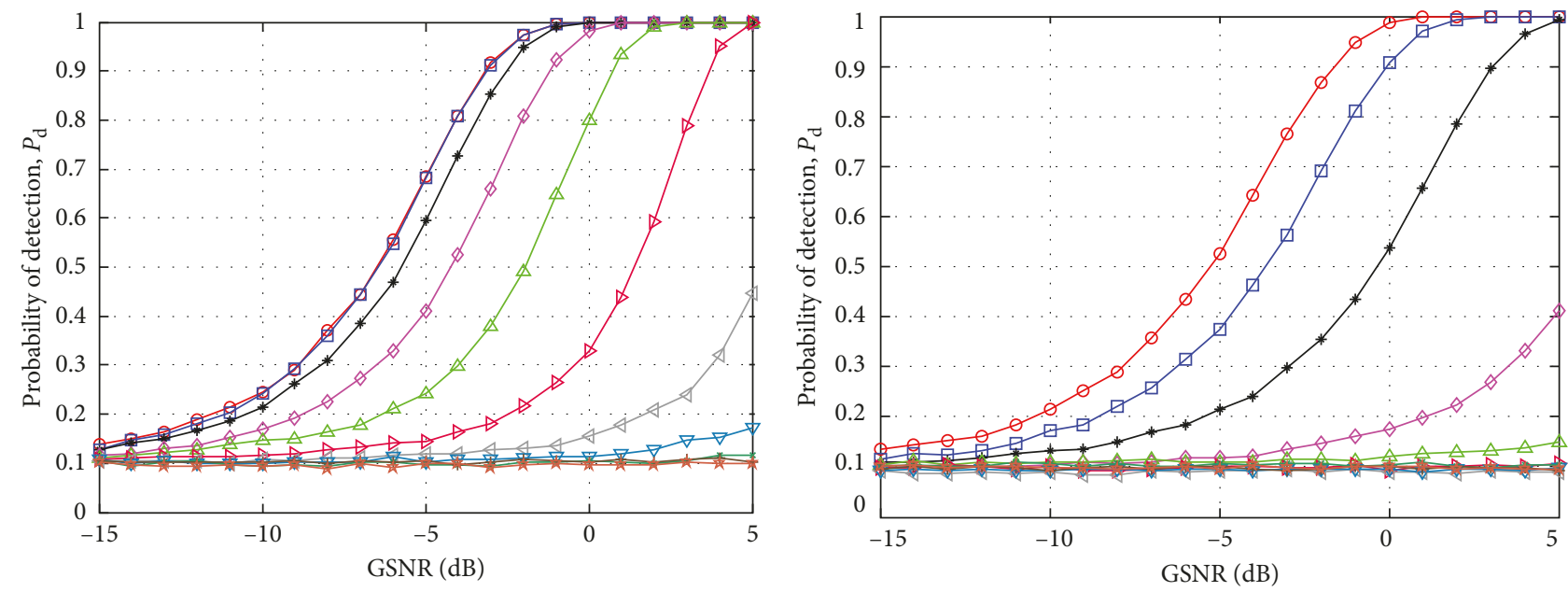

$$
\begin{array}{ll}
\rightarrow & \text { LM } \\
\square & \text { FLOM, } p=0.2 \\
\rightarrow & \text { FLOM, } p=0.4 \\
\multimap & \text { FLOM, } p=0.6 \\
\triangle & \text { FLOM, } p=0.8 \\
\rightarrow & \text { FLOM, } p=1
\end{array}
$$$$
\triangleleft \text { FLOM, } p=1.2
$$$$
\nabla \text { FLOM, } p=1.4
$$$$
\rightarrow \text { FLOM, } p=1.6
$$$$
\begin{aligned}
& +\quad \text { FLOM, } p=1.8 \\
& \star \quad \text { FLOM, } p=2
\end{aligned}
$$

$$
\begin{array}{ll}
\rightarrow & \text { LM } \\
\square & \text { FLOM, } p=0.2 \\
\rightarrow & \text { FLOM, } p=0.4 \\
\neg & \text { FLOM, } p=0.6 \\
\triangle & \text { FLOM, } p=0.8 \\
\neg & \text { FLOM, } p=1
\end{array}
$$

$$
\begin{aligned}
& \neg \text { FLOM, } p=1.2 \\
& \neg \quad \text { FLOM, } p=1.4 \\
& \neg \text { FLOM, } p=1.6 \\
& \neg \text { FLOM, } p=1.8 \\
& \text { FLOM, } p=2
\end{aligned}
$$

(a)

(b)



(c)

FIgURE 6: Detection probability versus GSNR for the logarithmic and FLOM detectors in nonfading channels, $\gamma=2, P_{\mathrm{f}}=0.1$, and $N=1000$. (a) $\alpha=1.5$; (b) $\alpha=1$; (c) $\alpha=0.5$.

logarithmic detector outperforms the Cauchy detector for different $\alpha$. In addition, the advantage of the logarithmic detector over the Cauchy detector becomes more obvious when $\alpha$ turns smaller. It indicates that the logarithmic detector is more suitable for detection in very impulsive noise than the Cauchy detector.

In Figure 6, the performance of the logarithmic detector is compared with the FLOM detector in nonfading channels, where $\gamma=2, P_{\mathrm{f}}=0.1, N=1000$, and $p \in\{0.2: 0.2: 2\}$. In Figure 6(a), when the order $p$ decreases from 2 to 0.2 , the detection probability of the FLOM detector becomes higher and approaches the performance of the logarithmic detector; the same phenomenon can be seen in Figure 6(b) and Figure 6(c). When $\alpha$ decreases, the FLOM detector shows a more serious performance degradation than the logarithmic detector, which proves that the logarithmic 




FIGURE 7: ROC of the logarithmic, FLOM, and Cauchy detectors in Rayleigh channels, $\gamma=2, N=1000$, and GSNR $=-5 \mathrm{~dB}$.

detector is more robust than the FLOM detector. Thus, the logarithmic detector performs better than the FLOM detector, and it is more suitable for signal detection in very impulsive noise.

Figure 7 shows the ROC curves of the logarithmic, FLOM, and Cauchy detectors for different values of $\alpha$ in Rayleigh channels. We can see that the logarithmic detector performs better than the FLOM and Cauchy detectors when $\alpha<1.5$. In addition, the advantage of the logarithmic detector over the FLOM and Cauchy detectors is much greater when $\alpha$ is smaller. Thus, whether the channel is nonfading or Rayleigh fading, the logarithmic detector can achieve better performance than the FLOM and Cauchy detectors, especially for small values of $\alpha$.

\section{Conclusion}

This paper studies the signal detection in impulsive noise modeled by $S \alpha S$ distribution. The logarithmic detector was proposed and the performance was analyzed. Simulation results show that the logarithmic detector performs better than the FLOM and Cauchy detectors in $S \alpha \mathrm{S}$ noise for small values of $\alpha$. In addition, the proposed detector is a nonparametric method, which makes it convenient in practical applications. Hence the logarithmic detector is a good alternative to the FLOM detector for signal detection in $S \alpha S$ impulsive noise.

\section{Appendix}

\section{A.1 The Mean and Variance of $\Lambda_{L M}$ under $H_{0}$}

If $Y$ is the logarithmic moment of a $S \alpha S$ random variable $Z$, namely, $Y=\log |Z|$, the mean and variance of $Y$ can be expressed as [12]

$$
\begin{aligned}
E(Y) & =C_{e}\left(\frac{1}{\alpha}-1\right)+\frac{1}{\alpha} \log \gamma, \\
\operatorname{Var}(Y) & =E\left\{(Y-E\{Y\})^{2}\right\}=\frac{\pi^{2}}{6}\left(\frac{1}{\alpha^{2}}+\frac{1}{2}\right),
\end{aligned}
$$

where $C_{e}=0.57721566 \ldots$ is the Euler constant.

Under $H_{0}$, the observation signal is

$$
z(n)=w(n) .
$$

So, $z(n)$ is a $S \alpha$ S random variable. Let $Y_{n}=\log [|z(n)|]$, then the mean of $\Lambda_{\mathrm{LM}}$ can be calculated as

$$
u_{0}=E\left[\frac{1}{N} \sum_{n=1}^{N} Y_{n}\right]=E\left[Y_{n}\right]=C_{e}\left(\frac{1}{\alpha}-1\right)+\frac{1}{\alpha} \log \gamma \text {. }
$$

And the variance can be obtained as

$$
\begin{aligned}
\sigma_{0}^{2} & =E\left[\left(\frac{1}{N} \sum_{n=1}^{N} Y_{n}\right)^{2}\right]-\left(E\left[\frac{1}{N} \sum_{n=1}^{N} Y_{n}\right]\right)^{2} \\
& =\frac{1}{N^{2}} \sum_{n=1}^{N}\left\{E\left[Y_{n}^{2}\right]-\left(E\left[Y_{n}\right]\right)^{2}\right\}=\frac{1}{N} * \frac{\pi^{2}}{6}\left(\frac{1}{\alpha^{2}}+\frac{1}{2}\right) .
\end{aligned}
$$

\section{A.2 The Mean and Variance of $\Lambda_{\mathrm{LM}}$ under $H_{1}$}

Under $H_{1}$, the observation signal is

$$
z(n)=h s(n)+w(n) .
$$

In order to calculate the mean and variance of $\Lambda_{\mathrm{LM}}$, we should firstly compute the first and second order moments of $Y=\log |Z|$. The logarithmic moments can be calculated from the FLOM as follows [13]:

$$
E\left[(\log |z|)^{n}\right]=\lim _{p \rightarrow 0} \frac{d}{d z^{n}} E\left[|z|^{p}\right] .
$$

To calculate $E\left[(\log |z|)^{n}\right]$, we should firstly compute $E\left[|z|^{p}\right]$ under $H_{1}$ as follows:

$$
E\left[|z|^{p}\right]=E\left[|z(n)|^{p}\right]=E\left[|h s(n)+w(n)|^{p}\right] .
$$

Note that $h$ remains constant during the detection period, so $h s(n)$ still obeys Gaussian distribution, namely, $h s(n) \sim N\left(0, h^{2} \sigma_{s}^{2}\right)$. Its characteristic function is $\Phi_{h s}(u)=$ $\exp \left(-\left(h^{2} \sigma_{s}^{2} / 2\right)|u|^{2}\right)$. Since the characteristic function of the sum of $n$ statistically independent random variables is equal to the product of the characteristic functions of the individual random variables [19], the characteristic function of $z(n)$ is obtained as

$$
\Phi_{z}(u)=\Phi_{h s}(u) \Phi_{w}(u)=\exp \left(-\gamma|u|^{\alpha}-\frac{h^{2} \sigma_{s}^{2}}{2}|u|^{2}\right) .
$$

Because $s(n)$ and $w(n)$ have symmetric probability density functions (PDFs), it is easy to prove that $z(n)$ also has symmetric PDF.

Then with a similar procedure to calculate $E|X|^{p}$ of a $S \alpha S$ random variable $X$ in [20], we can obtain 


$$
\begin{aligned}
E\left[|z|^{p}\right]= & \frac{1}{\Gamma(1-p) \cos ((\pi / 2) p)} \int_{0}^{\infty} \exp \left(-\gamma u^{\alpha}-\frac{h^{2} \sigma_{s}^{2}}{2} u^{2}\right) \\
& \times\left(\gamma \alpha u^{\alpha-p-1}+h^{2} \sigma_{s}^{2} u^{1-p}\right) d u .
\end{aligned}
$$

Substituting (A.10) into (A.7), we can obtain the first and second order moments of $Y=\log |Z|$. Let $E\left[|z|^{p}\right]=A(p) /$ $D(p)$, where $A(p)=\int_{0}^{\infty} \exp \left(-\gamma u^{\alpha}-\left(h^{2} \sigma_{s}^{2} / 2\right) u^{2}\right)\left(\gamma \alpha u^{\alpha-p-1}+\right.$ $\left.h^{2} \sigma_{s}^{2} u^{1-p}\right) d u$ and $D(p)=\Gamma(1-p) \cos ((\pi / 2) p)$. The first order moment of $Y$ is obtained as

$$
E[\log |z|]=\lim _{p \rightarrow 0} \frac{d E\left[|z|^{p}\right]}{d p}=\lim _{p \rightarrow 0} \frac{A^{\prime}(p) D(p)-A(p) D^{\prime}(p)}{D^{2}(p)},
$$

where

$$
\begin{aligned}
& A^{\prime}(p)=-\int_{0}^{\infty} E(u) \ln (u)\left(\gamma \alpha u^{\alpha-p-1}+h^{2} \sigma_{s}^{2} u^{1-p}\right) d u, \\
& D^{\prime}(p)=\Gamma^{\prime}(1-p) \cos \left(\frac{\pi}{2} p\right)-\frac{\pi}{2} \Gamma(1-p) \sin \left(\frac{\pi}{2} p\right) .
\end{aligned}
$$

Using the integral in [21], we can get

$$
\Gamma^{\prime}(1-p)=-\int_{0}^{\infty} x^{-p} e^{-x} \ln (x) d x=-\Gamma(1-p) \psi(1-p) .
$$

From the equation in [21], we have

$$
\psi(1)=-C_{e} .
$$

So,

$$
\lim _{p \rightarrow 0} \Gamma^{\prime}(1-p)=C_{e} .
$$

Then, the first order logarithmic moment is

$$
\begin{aligned}
E[\log |z|] & =\lim _{p \rightarrow 0} \frac{d E\left[|z|^{p}\right]}{d p} \\
& =-\int_{0}^{\infty} E(u) \ln (u) F(u) d u-C_{e} \int_{0}^{\infty} E(u) F(u) d u,
\end{aligned}
$$

where $E(u)$ and $F(u)$ are defined in (11) and (12).

The second order moment of $Y$ is obtained as

$$
\begin{aligned}
E\left[(\log |z|)^{2}\right] & =\lim _{p \rightarrow 0} \frac{d^{2} E\left[|z|^{p}\right]}{d p^{2}} \\
& =\lim _{p \rightarrow 0} \frac{d}{d p}\left[\frac{A^{\prime}(p) D(p)-A(p) D^{\prime}(p)}{D^{2}(p)}\right] .
\end{aligned}
$$

To solve the equation above, the key issue is to calculate $\lim _{p \rightarrow 0} D^{\prime \prime}(p)$ which should compute $\lim _{p \rightarrow 0} \Gamma^{\prime \prime}(1-p)$. From the equation in [21], we can obtain

$$
\begin{aligned}
\Gamma^{\prime \prime}(1-p) & =\int_{0}^{\infty} t^{-p} e^{-t}(\ln t)^{2} d t \\
& =\Gamma(1-p)\left\{\psi^{2}(1-p)+\zeta(2,1-p)\right\},
\end{aligned}
$$

where $\zeta(z, q)$ is defined as [21]

$$
\zeta(z, q)=\sum_{n=0}^{\infty} \frac{1}{(q+n)^{z}}, \quad[\operatorname{Re} z>1, q \neq 0,-1,-2, \ldots] .
$$

From the equation in [22], we know

$$
\zeta(2)=1+\frac{1}{2^{2}}+\frac{1}{3^{2}}+\cdots=\frac{\pi^{2}}{6} .
$$

So,

$$
\begin{aligned}
\lim _{p \rightarrow 0} \Gamma^{\prime \prime}(1-p) & =\lim _{p \rightarrow 0} \Gamma(1-p)\left\{\psi^{2}(1-p)+\zeta(2,1-p)\right\} \\
& =C^{2}+\frac{\pi^{2}}{6}, \\
\lim _{p \rightarrow 0} D^{\prime \prime}(p) & =C^{2}-\frac{\pi^{2}}{12} .
\end{aligned}
$$

Using the quotient rule, we can obtain

$$
\begin{aligned}
E\left[(\log |z|)^{2}\right]= & \lim _{p \rightarrow 0} \frac{d^{2} E\left[|z|^{p}\right]}{d p^{2}} \\
= & \int_{0}^{\infty} E(u)(\ln u)^{2} F(u) d u \\
& +2 C \int_{0}^{\infty} E(u) \ln u F(u) d u \\
& +\left(C^{2}+\frac{\pi^{2}}{12}\right) \int_{0}^{\infty} E(u) F(u) d u .
\end{aligned}
$$

After obtaining the first and second order moments of $Y=\log |Z|$, the mean and variance of $\Lambda_{\mathrm{LM}}$ can be easily obtained as follows:

$$
\begin{aligned}
u_{1} & =E\left\{\frac{1}{N} \sum_{n=1}^{N} Y_{n}\right\}=E[\log |z|], \\
\sigma_{1}^{2} & =E\left[\Lambda_{\mathrm{LM}}^{2}\right]-\left(E\left[\Lambda_{\mathrm{LM}}\right]\right)^{2} \\
& =\frac{1}{N^{2}}\left\{\sum_{n=1}^{N} E\left[Y_{n}^{2}\right]-\sum_{n=1}^{N}\left(E\left[Y_{n}\right]\right)^{2}\right\} \\
& =\frac{1}{N}\left\{E\left[(\log |z|)^{2}\right]-(E[\log |z|])^{2}\right\},
\end{aligned}
$$

where $E[\log |z|]$ and $E\left[(\log |z|)^{2}\right]$ are shown as (A.15) and (A.21), respectively.

\section{Conflicts of Interest}

The authors declare that there are no conflicts of interest regarding the publication of this manuscript.

\section{Acknowledgments}

This work is supported by the National Natural Science foundation of China (Project no. 61501484). 


\section{References}

[1] G. A. Tsihrintzis and C. L. Nikias, "Performance of optimum and suboptimum receivers in the presence of impulsive noise modeled as an alpha-stable process," IEEE Transactions on Communications, vol. 43, no. 2, pp. 904-914, 1995.

[2] M. Nassar, K. Gulati, A. K. Sujeeth, N. Aghasadeghi, B. L. Evans, and K. R. Tinsley, "Mitigating near-field interference in laptop embedded wireless transceivers," Journal of Signal Processing Systems, vol. 63, no. 1, pp. 1-12, 2011.

[3] C. L. Nikias and M. Shao, Signal Processing with Alpha-Stable Distributions and Applications, Wiley, Hoboken, NJ, USA, 1995.

[4] X. Yang and A. P. Petropulu, "Co-channel interference modeling and analysis in a Poisson field of interferers in wireless communications," IEEE Transactions on Signal Processing, vol. 51, no. 1, pp. 64-76, 2003.

[5] X. Kong and T. Qiu, "Adaptive estimation of latency change in evoked potentials by direct least mean p-norm time-delay estimation," IEEE Transactions on Biomedical Engineering, vol. 46, no. 8, pp. 994-1003, 1999.

[6] K. Hassan, R. Gautier, I. Dayoub, M. Berbineau, and E. Radoi, "Multiple-antenna-based blind spectrum sensing in the presence of impulsive noise," IEEE Transactions on Vehicular Technology, vol. 63, no. 5, pp. 2248-2257, 2014.

[7] E. E. Kuruoglu, W. Fitzgerald, and P. Rayner, "Near optimal detection of signals in impulsive noise modeled with a symmetric alpha-stable distribution," IEEE Communications Letters, vol. 2, no. 10, pp. 282-284, 1998.

[8] X. Li, J. Sun, S. Wang, L. Fan, and L. Chen, "Near-optimal detection with constant false alarm ratio in varying impulsive interference," IET Signal Processing, vol. 7, no. 9, pp. 824-832, 2013.

[9] H. G. Kang, I. Song, S. Yoon, and Y. H. Kim, "A class of spectrum-sensing schemes for cognitive radio under impulsive noise circumstances: structure and performance in nonfading and fading environments," IEEE Transactions on Vehicular Technology, vol. 59, no. 9, pp. 4322-4339, 2010.

[10] X. Zhu, W.-P. Zhu, and B. Champagne, "Spectrum sensing based on fractional lower order moments for cognitive radios in a-stable distributed noise," Signal Processing, vol. 111, pp. 94-105, 2015.

[11] V. M. Zolotarev, "Integral transfomations of distributions and estimates of parameters of multidimensional spherically symmetric stable laws," in Contribution to Probability: A Collection of Papers, E. Lukacs, J. Gani, and V. K. Rohatgi, Eds., pp. 283-305, New York Academic, New York, NY, USA, 1981.

[12] X. Ma and C. L. Nikias, "Parameter estimation and blind channel identification in impulsive signal environments," IEEE Transactions on Signal Processing, vol. 43, no. 12, pp. 2884-2897, 1995.

[13] E. E. Kuruoglu, "Density parameter estimation of skewedstable distributions," IEEE Transactions on Signal Processing, vol. 49, no. 10, pp. 2192-2201, 2001.

[14] J. Gonzalez, J. Paredes, and G. Arce, "Zero-order statistics: a mathematical framework for the processing and characterization of very impulsive signals," IEEE Transactions on Signal Processing, vol. 54, no. 10, pp. 3839-3851, 2006.

[15] R. Tandra and A. Sahai, "SNR walls for signal detection," IEEE Journal of Selected Topics in Signal Processing, vol. 2, no. 1, pp. 4-17, 2008.

[16] A. Mariani, A. Giorgetti, and M. Chiani, "Effects of noise power estimation on energy detection for cognitive radio applications," IEEE Transactions on Communications, vol. 59, no. 12, pp. 3410-3420, 2011.

[17] W. Yin, P. Ren, J. Cai, and Z. Su, "Performance of energy detector in the presence of noise uncertainty in cognitive radio networks," Wireless Networks, vol. 19, no. 5, pp. 629638, 2013

[18] A. Mohammadi, M. R. Taban, J. Abouei, and H. Torabi, "Cooperative spectrum sensing against noise uncertainty using Neyman-Pearson lemma on fuzzy hypothesis test," Applied Soft Computing, vol. 13, no. 7, pp. 3307-3313, 2013.

[19] J. G. Proakis, Digital Communications, The McGraw-Hill Companies Inc., New York, NY, USA, 4th edition, 2001.

[20] M. Shao and C. L. Nikias, "Signal processing with fractional lower order moments: stable processes and their applications," Proceedings of the IEEE, vol. 81, no. 7, pp. 986-1010, 1993.

[21] S. Gradshteyn and I. M. Ryzhik, Table of Integrals, Series, and Products, USA Academic, New York, NY, USA, 7th edition, 2007.

[22] M. Abramowitz and I. A. Stegun, Handbook of Mathematical Functions: with Formulas, Graphs, and Mathematical Tables, vol. 55, National Bureau of Standards, Washington, DC, USA, 1965. 


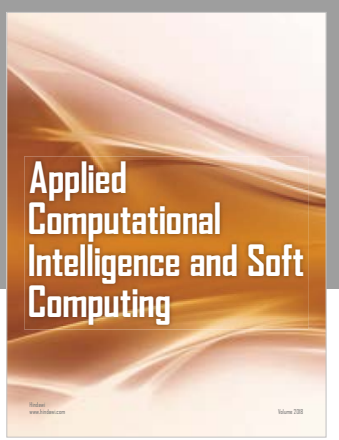



The Scientific World Journal
Submit your manuscripts at

Computing


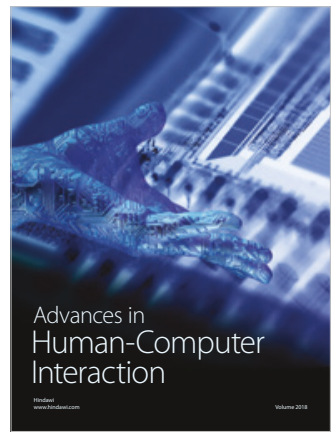

Human-Compute





Mathematical Problems in Engineering

\section{Engincering}
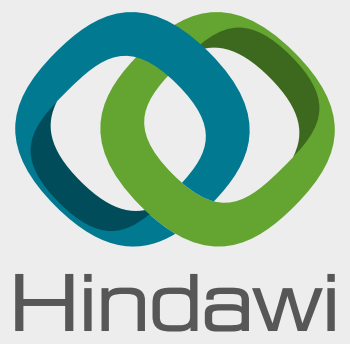

www.hindawi.com
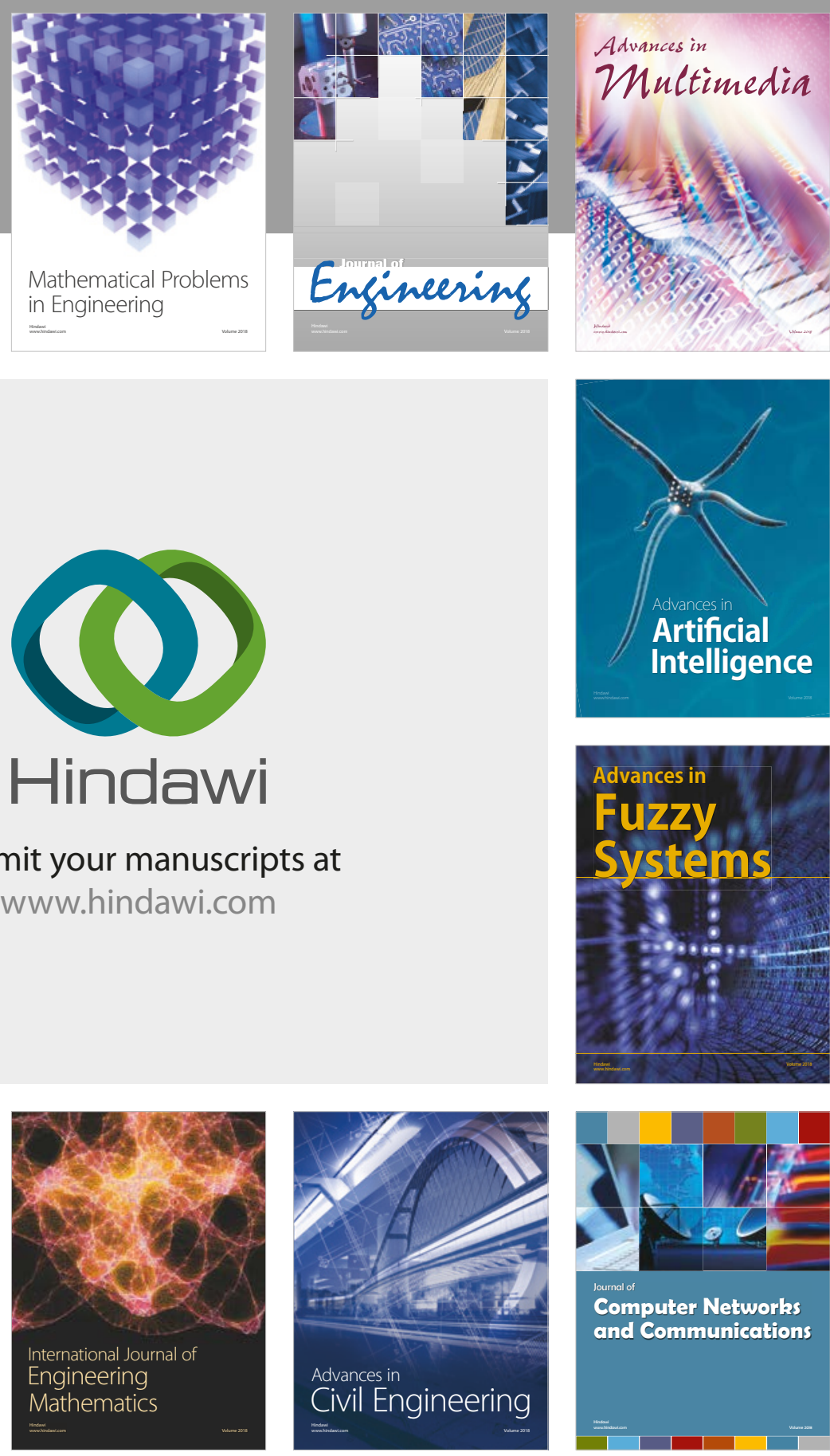

Computer Networks and Communications

Multimedia
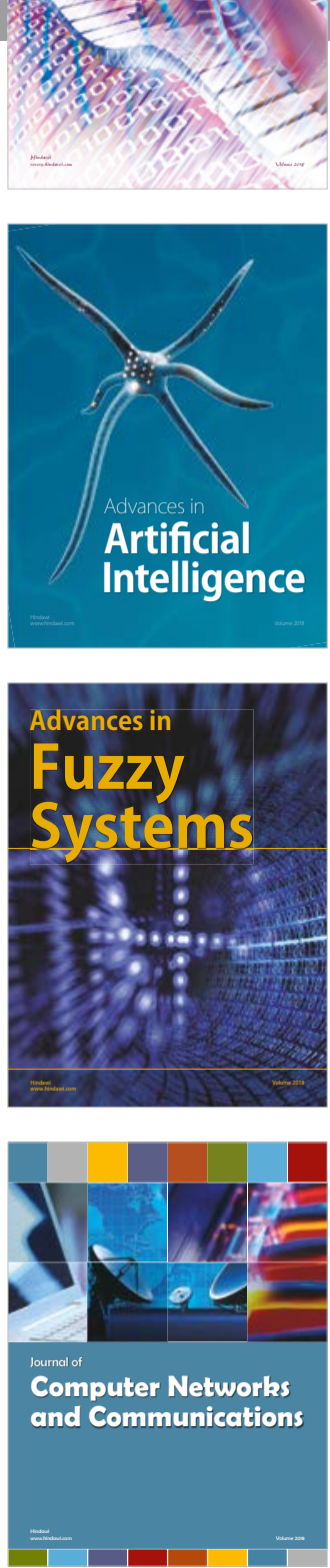

Advances in

Modelling \&

Simulation

in Engineering

interaction

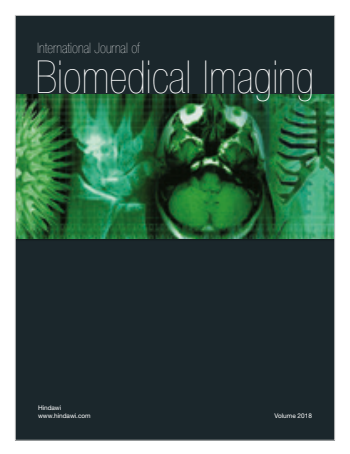

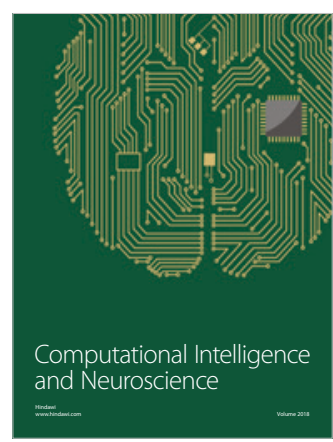

